\title{
Non-AIDS-defining cancer mortality among people with AIDS in Italy
}

\author{
Antonella Zucchetto ${ }^{1 *}$, Barbara Suligoi $^{2}$, Silvia Bruzzone ${ }^{3}$, Angela De Paoli ${ }^{1}$, Simona Pennazza ${ }^{2}$, Jerry Polesel ${ }^{1}$, \\ Luigino Dal Maso', Giovanni Rezza², Diego Serraino \\ From $12^{\text {th }}$ International Conference on Malignancies in AIDS and Other Acquired Immunodeficiencies \\ (ICMAOI) \\ Bethesda, MD, USA. 26-27 April, 2010
}

\section{Background}

Mortality due to non-AIDS-defining cancers (NADCs) among HIV-positive patients, in the era of highly active antiretroviral therapies, has yet to be completely defined. To estimate the excess risk of death for each NADC among people with AIDS (PWA), in comparison with the general population, we conducted a population-based study in Italy.

\section{Materials and methods}

Between 1999 and 2006, 10,391 Italian citizens (15 years of age) had been diagnosed with AIDS in Italy. These PWAs constituted the population included in our study. Death certificates were retrieved through a record-linkage with the mortality databases at the National Institute of Statistics. This allowed the extraction of information about PWA dates of death, up to December 31, 2006. The underlying cause of death, for each deceased PWA, was identified following the International Classification of Diseases, $10^{\text {th }}$ revision (ICD-10), applied also for the general population. The excess risks of death for each NADC were therefore estimated through standardized mortality ratios (SMRs) with 95\% confidence intervals (CIs), in comparison with the Italian general population.

\section{Results}

Among 3,209 deceased PWA, 7.4\% had died of NADCs, with an SMR of 6.6 (95\% CI 5.8-7.5). Significantly elevated excess risks of death were found for a broad range of cancers, notably for Hodgkin lymphoma (SMR=174), cancers of liver $(S M R=11.1)$, brain $(S M R=10.0)$, head and neck $(\mathrm{SMR}=8.2)$, lung $(\mathrm{SMR}=5.9)$, and myeloma and

\footnotetext{
*Correspondence: zucchetto.epi@cro.it

'Unit of Epidemiology and Biostatistics, National Cancer Institute "Centro di Riferimento Oncologico", Aviano, Italy

Full list of author information is available at the end of the article
}

leukemias (SMR=5.9). SMRs were higher among injecting drug users (IDUs) (SMR $=15.5$ for all NADC) than among other HIV-transmission categories $(\mathrm{SMR}=4.8)$, above all for liver cancer (SMR=65.2 among IDUs; $\mathrm{SMR}=2.8$ among non-IDUs).

\section{Conclusions}

We found particularly elevated SMRs for NADCs among PWA, with several excess risks of death that were higher than those expected, based on NADC incidence recorded in Italy [1]. This finding could be explained by the joint effect of an increased incidence of some malignancies, markedly those related to smoking and viral infections, and of their poorer prognosis among PWA versus the general population.

\section{Acknowledgements}

This article has been published as part of Infectious Agents and Cancer Volume 5 Supplement 1, 2010: Proceedings of the $12^{\text {th }}$ International Conference on Malignancies in AIDS and Other Acquired Immunodeficiencies (ICMAOI). The full contents of the supplement are available online at http://www.biomedcentral.com/1750-9378/5?issue=S1.

\section{Author details}

'Unit of Epidemiology and Biostatistics, National Cancer Institute "Centro di Riferimento Oncologico", Aviano, Italy. 'Department of Infectious Diseases, COA, National Institute of Health, Rome, Italy. ${ }^{3}$ Direzione centrale per le statistiche e le indagini sulle istituzioni sociali, Servizio Sanità e Assistenza, National Institute of Statistics, Rome, Italy.

Published: 11 October 2010

\section{Reference}

1. DalMaso L, Polesel J, Serraino D, et al: Pattern of cancer risk in persons with AIDS in Italy in the HAART era. Br J Cancer 2009, 100:840-847.

doi:10.1186/1750-9378-5-S1-A57

Cite this article as: Zucchetto et al:: Non-AIDS-defining cancer mortality among people with AIDS in Italy. Infectious Agents and Cancer 20105 (Suppl 1):A57. 